\title{
Extension of the line element-less method to dynamic problems
}

\author{
Antonina Pirrotta - Carsten Proppe
}

Received: date / Accepted: date

\begin{abstract}
The line element-less method is an efficient approach for the approximate solution of the Laplace or biharmonic equation on a general bidimensional domain. Introducing generalized harmonic polynomials as approximation functions, we extend the line element-less method to the inhomogeneous Helmholtz equation and to the eigenvalue problem for the Helmholtz equation. The obtained approximate solutions are critically discussed and advantages as well as limitations of the approach are pointed out.
\end{abstract}

\section{Introduction}

The line element-less method (LEM) is an innovative and efficient approach for torsion and bending analysis of beams and plates. The basic idea of the method is an expansion of the solution into harmonic polynomials and the determination of the expansion coefficients by minimizing a path integral in order to satisfy the boundary conditions. It can thus be considered as a Ritz method, where the approximation functions satisfy the partial differential equation in the interior of the domain. However, in contrast to boundary element methods, it is not necessary to subdivide the boundary into elements. For this reason, the solution can be very efficiently obtained by a semi-analytical procedure.

\footnotetext{
A. Pirrotta

Dipartimento di Ingegneria

Università degli studi di Palermo

Palermo 90128, Italy

E-mail: antonina.pirrotta@unipa.it

C. Proppe

Chair of Engineering Mechanics

Karlsruhe Institute of Technology (KIT)

76131 Karlsruhe, Germany

E-mail: carsten.proppe@kit.edu
} 
In this context LEM has been proposed for solving beam under torsion and shear $[8,9,15]$ without any discretization and evaluating line integrals only, even those used for performing the properties of cross-section as area, moment of inertia [14]. Further, based on the analogy between plates bending under edge moments and beams in torsion $[13,12,5]$, recently the aforementioned LEM has been employed for the bending problem of simply supported plates subject to uniformly distributed edge moments [16] and to different types of load [6].

Until now, the LEM is limited to problems that can be described by a Laplace or a biharmonic equation, as the harmonic polynomials are solutions of the Laplace equation. Thus, the LEM has been mainly applied in engineering to structural mechanics problems. In structural dynamics and acoustics, however, the solution of the Helmholtz equation is of utmost importance.

In this contribution, we present a first extension of the LEM to dynamic problems. To this end, we consider the application of the LEM to the Helmholtz equation on a bidimensional domain with Dirichlet boundary conditions:

$$
\Delta u+k^{2} u=q(x, y) \text { on } \Omega, u=0 \text { on } \partial \Omega
$$

We study two scenarios:

1. The computation of solutions for the inhomogeneous Helmholtz equation.

2. The eigenvalue problem for the homogeneous Helmholtz equation.

In order to apply the LEM to the Helmholtz equation, it is necessary to introduce a new set of approximation functions. These approximation functions are obtained by a transformation that maps solutions of the Laplace equation to those of the Helmholtz equation. The new approximation functions can be best represented in polar coordinates and involve products of harmonic functions and Bessel functions of first kind. With these approximation functions, it is then still possible to solve the Helmholtz equation by a semi-analytical approach, without the necessity to subdivide the boundary into elements.

The new approach is presented and discussed with several examples, which illustrate the efficiency of the method. Moreover, convergence studies are presented.

The paper is organized as follows: In the next section, the line elementless method for the bidimensional Laplace equation is briefly described and compared to other methods. After this, a general approach is introduced that allows to obtain approximation functions for the Helmholtz equation and even for general elliptic equations. These approximation functions are then combined with the LEM in order to obtain approximate solutions for the inhomogeneous Helmholtz equation and in order to solve the eigenvalue problem for the homogeneous Helmholtz equation on general bidimensional domains. The obtained solutions are critically discussed. The last section contains conclusions and an outlook on future applications of the LEM. 


\section{Brief outline of the line element-less method}

Consider the nonhomogeneous bidimensional Laplace equation on a simply connected domain $\Omega$ :

$$
\Delta u=q(x, y) \text { on } \Omega, u(x, y)=0 \text { on } \partial \Omega .
$$

with Dirichlet boundary condition

$$
u(x, y)=0 \text { on } \partial \Omega .
$$

For the LEM, the solution is expanded as follows [6]:

$$
u(x, y)=\sum_{i=0}^{n} a_{i} P_{i}(x, y)+\sum_{j=1}^{n} b_{j} Q_{j}(x, y)+u_{p}(x, y),
$$

where

$$
\begin{aligned}
P_{i}(x, y) & =\operatorname{Re}(x+\imath y)^{i} \\
Q_{j}(x, y) & =\operatorname{Im}(x+\imath y)^{j}
\end{aligned}
$$

are the harmonic polynomials and $u_{p}(x, y)$ represents the particular solution. The expansion coefficients are obtained from a variational formulation for the boundary condition:

$$
\partial \oint_{\Gamma}(u(x, y))^{2} \mathrm{~d} \Gamma=0
$$

where $\Gamma$ denotes the contour curve of $\Omega$. The integrand is the square difference between the solution $u(x, y)$ and the boundary function given by eq. (3) and the variation is thus carried out on all functions that satisfy the Laplace equation (2). Inserting the series expansion (4) into the variational formulation (6) yields the following equation

$$
\partial J(\mathbf{a}, \mathbf{b})=0,
$$

for the series expansion coefficients that are assembled in the vectors $\mathbf{a}, \mathbf{b}$. Equation (7) leads to a linear algebraic problem for the expansion coefficients.

The LEM differs in the variational formulation eq. (7) from other methods and it is this formulation that leads to a truly element-less approach. E.g in the complex polynomial method [11], the expansion

$$
u(x, y)=\sum_{i=0}^{n}\left(a_{i}+\imath b_{i}\right)(x+\imath y)^{i}+u_{p}(x, y)
$$

is the same as in eq. (4), but the expansion coefficients are determined at $n+1$ points of the boundary, thus by collocation. In the interior of the domain, one then finds

$$
u(z)=\frac{1}{2 \pi \imath} \oint_{\Gamma} \frac{u(\zeta)}{\zeta-z} \mathrm{~d} \Gamma
$$


where $z=x+\imath y$. In the complex variable boundary element method $[10,2,1$, 4], a linear interpolant $G(\zeta)$ is constructed on the boundary. In the interior of the domain, the solution is determined from

$$
u(z)=\frac{1}{2 \pi \imath} \oint_{\Gamma} \frac{G(\zeta)}{\zeta-z} \mathrm{~d} \Gamma .
$$

In [3], the authors show how competitive the LEM is in comparison with the complex polynomial method $(\mathrm{CPM})$ and the complex variable boundary method (CVBEM). Especially regarding the robustness of the method related to the adivisability to capture exact solutions (when available) only retaining a few series coefficients and exactly satisfying the boundary condition continuously.

\section{Generalized harmonic polynomials}

Vekua [17] and Bergman [7] developed independently a theory to obtain solutions for the generalized elliptic equation

$$
\Delta u+a(x, y) u_{x}+b(x, y) u_{y}+c(x, y) u=0,
$$

where $u_{x}$ and $u_{y}$ denote the partial derivatives of $u(x, y)$ with respect to the first and second argument, by mapping holomorphic functions on solutions of (11). Define

$$
\begin{aligned}
& 4 A(z, \zeta)=a\left(\frac{z+\zeta}{2}, \frac{z-\zeta}{2 \imath}\right)+\imath b\left(\frac{z+\zeta}{2}, \frac{z-\zeta}{2 \imath}\right), \\
& 4 B(z, \zeta)=a\left(\frac{z+\zeta}{2}, \frac{z-\zeta}{2 \imath}\right)-\imath b\left(\frac{z+\zeta}{2}, \frac{z-\zeta}{2 \imath}\right), \\
& 4 C(z, \zeta)=c\left(\frac{z+\zeta}{2}, \frac{z-\zeta}{2 \imath}\right) .
\end{aligned}
$$

The Riemann function $G(z, \zeta, t, \tau)$ is given by

$$
\begin{gathered}
G(z, \zeta, t, \tau)=1+\int_{\tau}^{\zeta} A(z, \eta) G(z, \eta, t, \tau) \mathrm{d} \eta+\int_{t}^{z} B(\xi, \zeta) G(\xi, \zeta, t, \tau) \mathrm{d} \xi \\
-\int_{t}^{z} \int_{\tau}^{\zeta} C(\xi, \eta) G(\xi, \eta, t, \tau) \mathrm{d} \xi \mathrm{d} \eta
\end{gathered}
$$

and allows to represent the solution of eq. (11) for any holomorphic function $\phi(z)$ by

$$
u(x, y)=\operatorname{Re}\left(G\left(z, \bar{z}_{0}, z, \bar{z}\right) \phi(z)+\int_{z_{0}}^{z} \phi(t) H\left(t, \bar{z}_{0}, z, \bar{z}\right) \mathrm{d} t\right),
$$

where $z_{0}$ fixed. The function $H(z, \zeta, t, \tau)$ is given by

$$
H(z, \zeta, t, \tau)=B(z, \zeta) G(z, \zeta, t, \tau)-\frac{\partial G}{\partial z}(z, \zeta, t, \tau)
$$


and can thus be computed from $B(z, \zeta)$ and $G(z, \zeta, t, \tau)$. The solution is thus completely determined by the Riemann function $G(z, \zeta, t, \tau)$ which is governed by a Volterra integral equation. For special cases of eq. (13), closed form expressions for the Riemann function are available. E.g., for the Laplace equation, one has $G=1$ and for the Helmholtz equation $G(z, \zeta, t, \tau)=$ $J_{0}(k \sqrt{(z-t)(\zeta-\tau)})$.

In order to generate the approximation functions that satisfy the differential equation (11), we set $\phi(z)$ to the harmonic polynomials:

$$
\phi(z)=1, z, \imath z, z^{2}, \imath z^{2} \ldots
$$

Taking the real and imaginary part of (14) with $\phi(z)$ given by $(16)$ and $z_{0}=0$, we obtain for the Helmholtz equation the families of approximation functions

$$
\begin{aligned}
P_{i}(x, y) & =\cos (i \theta) J_{i}(k r), \\
Q_{i}(x, y) & =\sin (i \theta) J_{i}(k r),
\end{aligned}
$$

where $z=x+\imath y=r \exp (i \theta)$ and $J_{i}(k r)$ is the Bessel function of first kind of order $i$.

\section{Line element-less method for the nonhomogeneous Helmholtz equation}

Consider the nonhomogeneous bidimensional Helmholtz equation on a simply connected domain $\Omega$ with Dirichlet boundary conditions:

$$
\Delta u+k^{2} u=q(x, y) \text { on } \Omega, u=0 \text { on } \partial \Omega .
$$

Following the theory of Bergman and Vekua [7,17] on generalized harmonic polynomials, the expansion for the LEM is now written as

$$
u(x, y)=\sum_{i=0}^{n} a_{i} P_{i}(x, y)+\sum_{i=1}^{n} b_{i} Q_{i}(x, y)+u_{p}(x, y)
$$

where the approximation functions $P_{i}(x, y), Q_{i}(x, y), i=1, \ldots, n$, are given by (17). As in the LEM for the Laplace equation, the expansion coefficients are obtained from the variational formulation

$$
\partial \oint_{\Gamma}(u(x, y))^{2} \mathrm{~d} \Gamma=0,
$$

that is discretized by inserting the series expansion (19) to

$$
\partial J(\mathbf{a}, \mathbf{b})=0,
$$

where $\mathbf{a}, \mathbf{b}$ assemble the expansion coefficients.

Example 1: Consider the Helmholtz equation

$$
\Delta u+4 u=-16-16\left(x^{2}+y^{2}\right) \text { on } \Omega,
$$


a)

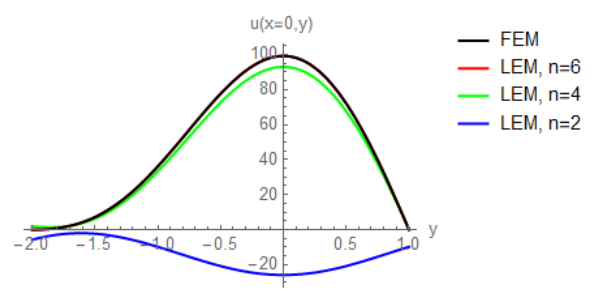

b)

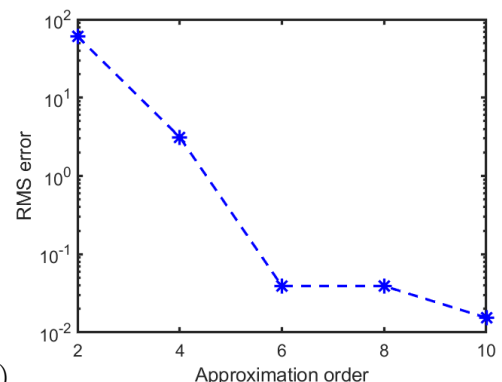

Fig. 1 a) Approximate solution $u(x=0, y)$ of the nonhomogeneous Helmholtz equation on a triangular domain. b) Dependence of the root mean square error between the FEM solution and the LEM solution on the approximation order $n$.

Table 1 Numerical results for $u(x, y)$ at selected points $(x, y)$ in the interior of the solution domain.

\begin{tabular}{cccc}
\hline Approximation order $n$ & $x=0 ., y=0.5$ & $x=0 ., y=-1$. & $x=1 ., y=0.5$ \\
\hline 2 & -20.753 & -9.7796 & -7.3337 \\
4 & 67.6100 & 33.2529 & 24.5642 \\
6 & 72.1596 & 36.0978 & 26.9297 \\
8 & 72.1596 & 36.0978 & 26.9297 \\
10 & 72.1453 & 36.1187 & 26.9481 \\
FEM & 72.1346 & 36.1083 & 26.9371 \\
\hline
\end{tabular}

where $\Omega$ is a triangular domain given by the vertices $(x=0, y=-2)$, $(x=\sqrt{3}, y=1),.(x=-\sqrt{3}, y=1$.). Figure 1a) displays graphs of the approximate solutions $u(x=0, y)$ for eq. (22) obtained with the finite element method (FEM) and with LEM and approximation orders of $n=2, n=4$ and $n=6$, respectively. The FEM solution has been obtained with a mesh of 320 linear traingular elements and 685 nodes. It can be seen that the LEM solution converges quickly to the FEM solution. In fact, the FEM solution and the LEM solution of order $n=6$ cannot be distinguished. Moreover, Figure 1 b) demonstrates that the root mean square error between the FEM and the LEM solution decreases exponentially with the approximation order $n$. This finding is also confirmed from evaluations of the numerical solutions at selected points, cf. Table 1. Starting with the approximation order $n=6$, the solution obtained with the LEM corresponds very well to the finite element solution; moreover, there is no difference between approximation order 6 and 8 of the LEM. For these pointwise evaluations, it can be seen from Table 1 that the FEM solution is underestimated in some cases and overestimated in others. 
Table 2 First three eigenvalues computed with a direct formulation.

\begin{tabular}{cccc}
\hline Approximation order $n$ & Mode $k_{1}$ & Mode $k_{2}$ & Mode $k_{3}$ \\
\hline 1 & 2.40482 & 3.8333 & - \\
2 & 2.40482 & 3.8333 & 5.13561 \\
3 & 2.40482 & 3.8333 & 5.13561 \\
\hline
\end{tabular}

\section{Line element-less method for the homogeneous Helmholtz equation}

Consider the homogeneous bidimensional Helmholtz equation on a simply connected domain $\Omega$ with Dirichlet boundary conditions:

$$
\Delta u+k^{2} u=0 \text { on } \Omega, u=0 \text { on } \partial \Omega .
$$

In order to compute the eigenvalue $k$ by the LEM, a direct approximation of the solution by

$$
u(x, y)=\sum_{i=0}^{n} a_{i} P_{i}(x, y)+\sum_{i=1}^{n} b_{i} Q_{i}(x, y)
$$

with approximation functions $P_{i}(x, y), Q_{i}(x, y), i=1, \ldots, n$, given by (17) is considered. In this case, the variational formulation eq. (21) for the Dirichlet boundary condition leads to the linear algebraic system $A_{k} \mathbf{c}=\mathbf{0}$, with $\mathbf{c}=$ $[\mathbf{a} \mathbf{b}]^{T}$, from which the characteristic equation $\operatorname{det}\left(A_{k}\right)=0$ is obtained. Note however, that this characteristic equation equation is not a polynomial in $k$. In general, the nonlinear characteristic equation for the eigenvalue $k$ can only by solved by iterative methods.

Example 2: Consider the homogeneous Helmholtz equation eq. (23) on the unit circle with Dirichlet boundary conditions. For this case, the first three eigenvalues are obtained as roots of the Bessel functions of the first kind: $k_{1}=2.40483, k_{2}=3.83170, k_{3}=5.13562$. Table 2 summarizes the results computed for three different aproximation orders $(n=1, n=2, n=3)$. As the direct approximation is a linear combination of the eigenfunctions, the approximation quality is in general very good and independent on the approximation order. In fact, the deviations between the theoretical eigenvalues and the obtained ones may be attributed to numerical errors. However, due to the nonlinearity of the characteristic equation, it was not possible to obtain convergence to all eigenvalues. In contrast, convergence to a specific eigenvalue seems to depend on the approximation order. Moreover, many iterations were necessary until convergence and spurious modes were found.

In order to overcome these difficulties, it is necessary to formulate the problem as a linear eigenvalue problem for which the numerical solution methods 
Table 3 First two eigenvalues computed with a power series expansion.

\begin{tabular}{ccc}
\hline$n$ & $N$ & $k_{1}$ \\
\hline 1 & 1 & 1.41 \\
1 & 2 & 2. \\
1 & 3 & 1.91 \\
1 & 4 & 2.65 \\
\hline
\end{tabular}

\begin{tabular}{ccc}
\hline$n$ & $N$ & $k_{1}$ \\
\hline 2 & 1 & - \\
2 & 2 & 2. \\
2 & 3 & 2.45 \\
2 & 4 & 2.65 \\
\hline
\end{tabular}

\begin{tabular}{cccc}
\hline$n$ & $N$ & $k_{1}$ & $k_{2}$ \\
\hline 3 & 1 & - & - \\
3 & 2 & - & - \\
3 & 3 & 2.45 & - \\
3 & 4 & 2.65 & 2.83 \\
\hline
\end{tabular}

developed for matrix eigenvalue problems can be applied. To this end, a truncated power series expansion of the approximation given by

$$
u(x, y)=\sum_{i=0}^{n} a_{i} P_{i}(x, y)+\sum_{i=1}^{n} b_{i} Q_{i}(x, y)
$$

with

$$
\begin{aligned}
P_{i}(x, y) & =\cos (i \theta) \sum_{m=0}^{N} \frac{(-1)^{m} k^{2 m+i}\left(\frac{r}{2}\right)^{2 m+i}}{(i+m) ! m !} \\
Q_{i}(x, y) & =\sin (i \theta) \sum_{m=0}^{N} \frac{(-1)^{m} k^{2 m+i}\left(\frac{r}{2}\right)^{2 m+i}}{(i+m) ! m !}
\end{aligned}
$$

is considered. The variational formulation eq. (21) leads then to

$$
\left(\sum_{m=0}^{2 N} k^{m} A_{m}\right) \mathbf{c}=\mathbf{0},
$$

where the coefficient matrix is now expressed in terms of powers of the eigenvalue $k$. Eq. (27) can be reformulated as the linear eigenvalue problem

$$
\left[\begin{array}{ccccc}
A_{0} & A_{1} & A_{2} & \ldots & A_{2 N-1} \\
0 & I & \ldots & & \\
& & \ddots & & \\
& & & & I
\end{array}\right]\left[\begin{array}{c}
\mathbf{c} \\
k \mathbf{c} \\
\vdots \\
k^{2 N-1} \mathbf{c}
\end{array}\right]=k\left[\begin{array}{ccccc}
0 & 0 & 0 & \ldots & -A_{2 N} \\
0 & I & \ldots & & \\
& \ddots & & \\
& & & & I
\end{array}\right]\left[\begin{array}{c}
\mathbf{c} \\
k \mathbf{c} \\
\vdots \\
k^{2 N-1} \mathbf{c}
\end{array}\right]
$$

which can now be solved for the eigenvalues $k$ by numerical methods for linear eigenvalue problems.

Example 3: Consider again the homogeneous Helmholtz equation (23) on the unit circle with Dirichlet boundary conditions. Table 3 summarizes the results for three different approximation orders $n$ and a power series expansion up to fourth order. It can be seen that the approximation error is quite large and that a very high expansion order is necessary in order to achieve convergence. 


\section{Conclusions}

The LEM is a boundary Ritz method that does not require any discretization. As shown in this study, the method can be applied not only to Laplace and biharmonic equations, but also to general linear elliptic equations by an appropriate choice of the approximation functions. However, in the more general case, a numerical integration might be required in order to determine the coefficient matrix of the linear system that governs the expansion coefficients.

While the results for the nonhomogeneous Helmholtz equation are quite encouraging, the solution of the eigenvalue problem is challenging, since the coefficient matrix is a nonlinear function of the free parameter. Neither the direct formulation nor a power series expansion seem to be adequate for solving the eigenvalue problem.

In summary, the LEM is an efficient solution method for elliptic equations on general bidimensional domains. In this regard, possible applications comprise fast and efficient homogenization methods in continuum mechanics. Regarding the eigenvalue problem, a combination of the direct formulation and the series expansion as well as a computation of the gradient might help to overcome the convergence problems.

\section{Compliance with Ethical Standards}

Funding: The first author acknowledges financial support PRIN2015, 2015JW9NJT_001: Advanced mechanical modeling of new materials and structures for the solution of 2020 Horizon challenges.

Conflict of Interest: The authors declare that they have no conflict of interest.

\section{References}

1. Barone, G., Pirrotta, A.: CVBEM application to a novel potential function providing stress field and twist rotation at once. Journal of Engineering Mechanics 139(9), 12901293 (2013)

2. Barone, G., Pirrotta, A.: CVBEM for solving De Saint-Venant solid under shear forces. Engineering Analysis with Boundary Elements 37, 197-204 (2013)

3. Barone, G., Pirrotta, A., Santoro, R.: Comparison among three boundary element methods for torsion problems: CPM, CVBEM, LEM. Engineering Analysis with Boundary Elements 35, 895-907 (2011)

4. Barone, G., Pirrotta, A., Santoro, R.: CVBEM solution for de saint-venant orthotropic beams under coupled bending and torsion. Acta Mechanica 226(3), 783-796 (2015)

5. Barretta, R.: Analogies between Kirchhoff plates and Saint-Venant beams. Acta Mechanica 224, 2955-2964 (2013)

6. Battaglia, G., Di Matteo, A., Micale, G., Pirrotta, A.: Arbitrarily shaped plates analysis via Line Element-Less Method (LEM). Thin-Walled Structures 133, 235-248 (2018)

7. Bergman, S.: Integral Operators in the Theory of Linear Partial Differential Equations. Springer (1961)

8. Di Paola, M., Pirrotta, A., Santoro, R.: Line element-less method (LEM) for beam torsion solution (truly no-mesh method). Acta Mechanica 195, 349-364 (2008) 
9. Di Paola, M., Pirrotta, A., Santoro, R.: De Saint-Venant flexure-torsion problem handled by Line Element-less Method (LEM). Acta Mechanica 217, 101-118 (2011)

10. Hromadka II, T.V.: The complex variable boundary element method. Springer, Heidelberg, New York, Tokyo (1984)

11. Hromadka II, T.V., Guymon, L.G.: Complex polynomial approximation of the laplace equation. Journal of Hydraulic Engineering 110(3), 329-339 (1984)

12. Irschik, H.: Analogies between bending of plates and torsion problem. J. Eng. Mech. 117, 2503-2508 (1991)

13. Murtha-Smith, E.: Plate analogy for the torsion problem. J. Eng. Mech. 116, 1-17 (1990)

14. Pirrotta, A.: Complex potential function in elasticity theory: shear and torsion solution through line integrals. Acta Mechanica 223(6), 1251-1259 (2012)

15. Pirrotta, A.: LEM for twisted re-entrant angle sections. Computers and Structures 133, 149-155 (2014)

16. Pirrotta, A., Bucher, C.: Innovative straight formulation for plate in bending. Computers and Structures 180, 117-124 (2017)

17. Vekua, I.N.: New Methods for Solving Elliptic Equations. North Holland (1967) 